\title{
Optimal Network Reconfiguration with Distributed Generation and Electric Vehicle Charging Stations
}

\author{
Surender Reddy Salkuti \\ Department of Railroad Electrical Systems, \\ Woosong University, Daejeon, 34606, Republic of Korea. \\ Corresponding author: surender@wsu.ac.kr
}

(Received on March 07, 2021; Accepted on June 27, 2021)

\begin{abstract}
This paper proposes an optimal network reconfiguration (ONR) by integrating the renewable energy (RE) based distributed generation (DG) resources, i.e., wind and solar photovoltaic (PV) modules, and electric vehicle charging stations (EVCS). The uncertainties related to renewable energy sources (RESs) are handled by using probability analysis. In this work, wind uncertainty is handled by using Weibull probability density function (PDF), and solar PV uncertainty is modeled by using Beta PDF. This paper also models the load of EVCSs. The ONR is a tool to operate distribution systems (DSs) at optimum cost/loss. In the literature, most of the ONR problems are solved as single objective type. This neccessiate the development of multi-objective based ONR problem and solved using the multiobjective algorithms by considering multiple objectives. Therefore in this paper, total cost of operation and power losses are considered as two objectives functions. The single objective-based ONR is solved using crow search algorithm (CSA) and multi-objective-based ONR is solved using multi-objective-based CSA. As the DS is unbalanced, the power flow for the unbalanced system will include the three-phase transformer. The ONR problem has been solved by considering 17 bus unbalanced and balanced DSs.
\end{abstract}

Keywords- Electric vehicles, Distribution system, Network reconfiguration, Renewable energy, Uncertainty.

\section{Introduction}

The rapid expansion of power transmission and distribution systems due to exponential load growth leads to poor voltage regulation and higher power losses. To meet this demand at reasonal cost, power sectors have adopted remarkable modifications towards power sector. The investment on the modification or upgradation of the existing network is practically a hard challenge because of strict economical and environmental constraints. This causes the distribution systems (DSs) to operate closer to their voltage stability boundaries, which makes the operation and control of distribution sytsems further complicated. So, the present focus of power utilities to use the existing resources in an optimal way. Feeder reconfiguration (FRC)/ONR is a very important tool for the DSs to operate at optimum cost and system losses and to improve system's reliability/ security. Deregulation of energy market, restructuring of utilities, technological developments and global environmental concerns, have increased the interest of power utilities in the use of sustainable RESs (Pamshetti et al., 2020). In last few years many research attempts are made for the integration of renewable generation together with ONR to achieve the objectives of power loss, cost minimization along with maximization of reliability, economic and environmental benefits. By operating the radial DS at its current capacity limits rather than voltage limits can enhance the reliability of the system (Nick et al., 2018). However, the efficiency can be increased by reducing the losses by optimal FRC, shunt capacitors, etc. Advantages of ONR include better voltage profile, balancing the loading among the feeders, minimize the total cost and power losses, and relieve current limit violation. Cost/ loss minimization in the DS can be obtained by using the FRC, i.e., by changing the feeder topology by opening/closing status of sectionalizing and tie 
switches. A repeated load flow solution is required for this FRC (Kavousi-Fard et al., 2015).

A two-stage solution methodology integrating the coordinated electric vehicle (EV) charging with ONR to optimize the performance of DS has been proposed in (Amin et al., 2020). Naidu et al. (2019) proposes a particle swarm optimization (PSO) technique for the charging of EV with FRC to charge a higher number of EVs and enhance the system performance. Smart charging/discharging of plug-in EVs and demand response programs for enhancing the reliability of radial DSs using PSO have been proposed in Sadeghian et al. (2019). A multi-objective-based optimal approach with coordinated scheduling of EVs into the building cluster for enhancing the economical and safe operation in the DSs has been proposed in Huang et al. (2020). Mozafar et al. (2017) proposes an approach for optimal sizing and location of renewable energy sources (RESs) and EV charging stations (EVCS) using genetic algorithm (GA)-PSO-based hybrid optimization algorithm. Optimal locations of EVCSs considering the optimization of voltage deviation, power loss and maximization of voltage stability index is proposed in Babu and Swarnasri (2020). An approach to enhance the hosting capacity of DSs at EVCSs with reliability-based ONR has been described in Kamruzzaman et al. (2020).

Kavousi-Fard et al. (2015) proposes a stochastic approach with an unscented transformation to handle the uncertain behavior of EVs based on the correlated power generation of WEGs. An ONR problem of DSs with an unbalanced optimal power flow (OPF) approach addressing the operational characteristics of an unbalanced DS, RESs, and voltage regulators has been proposed in Liu et al. (2019). Fu and Chiang (2018) proposes a four-stage solution approach including the assessment, evaluation and optimization stages for solving the problem of large-scale nonlinear mixed-integer optimization. Wu et al. (2020) proposes an ONR of DSs to handle the intermittances due to solar PV, wind powers, and load and optimizing voltage stability and power loss minimization. Hourly and whole-day ONR has been performed by modeling the solar irradiance, wind speed, and load forecasting using wavelet transform-based ARIMA model (Gangwar et al., 2020). An optimal stochastic FRC approach has been proposed in Rostami et al. (2015) to analyze the charging behavior of EVs by varying the grid topology. An ONR problem is solved in Asrari et al. (2016) using a hybrid optimization technique by combining the concept of fuzzy-based Pareto dominance with evolutionary-based shuffled frog leaping technique. An approach for an optimal allocation of storage systems, wind energy generators (WEGs), EVs and demand response, and hourly ONR in normal and contingency situations has been proposed in Kianmehr et al. (2019). An optimum power ow (OPF) based heuristic approach for ONR has been formulated in Peng et al. (2015), where continuous functions are used to model open/close the switch. The proposed technique starts by closing all the switches. Then based on the heuristic technique obtain from OPF solution the switch that candidate to be open to break the next loop is selected. This process is iterated until all the radial configuration structured is obtained.

From the literature, it is clear that the ONR/FRC problem is solved individually or jointly by considering the RESs and capacitor banks. However, EVCSs are not considered. Therefore, the objective of present paper is to perform an optimal FRC by integrating RESs and EVCSs. This work quantifies the techno-economic benefits of ONR/FRC in the presence of RESs and EVCSs. Total cost and power losses are considered as two important and conflicting objectives and they are solved independently and simultaneously using crow search algorithm (CSA) and multiobjective CSA (MO-CSA), respectively. 
The rest of the paper will be broken into the following parts: modeling of RESs and EVCSs has been presented in section 2. The mathematical modeling of proposed ONR is described in section 3. Section 4 presents simulation results of ONR problem on balanced and unbalanced 17 bus DSs. Lastly, the concluding remarks and contributions are set out in Section 5.

\section{Modeling of System Components}

This section presents the modeling of RESs, i.e., WEGs, solar PV modules, and electric vehicle charging stations (EVCSs).

\subsection{Modeling of WEGs}

Power generated from the WEG depends on geographical location, wind speed $(v)$, and direction Koutsoukis et al. (2017). However, for calculating the amount of power from the WEG, in this paper, only the wind speed $(v)$ is considered, and it is modeled as Li et al. (2017).

$P_{W}=\left\{\begin{array}{lr}0 & v \leq v_{\text {cin }}, v>v_{\text {cout }} \\ \left(\frac{P_{W}^{r}}{v_{r}^{3}-v_{\text {cin }}^{3}}\right) v^{3}-\left(\frac{v_{\text {cin }}^{3}}{v_{r}^{3}-v_{\text {cin }}^{3}}\right) P_{\text {rated }} & v_{\text {cin }}<v \leq v_{r} \\ P_{W}^{r} & v_{r}<v \leq v_{\text {cout }}\end{array}\right.$

For the modeling of wind power uncertainty, a two-parameter Weibull probability distribution function (PDF) is used in this paper, which is more accurate for the modeling of wind speed. The cumulative distribution function $\left(F_{W}(v)\right)$ of wind speed $(v)$ is represented by Jung and Salkuti (2020).

$F_{W}(v)=1-e^{-\left(\frac{v}{c}\right)^{k}}$

$\mathrm{c}$ and $\mathrm{k}$ are scalar and shape parameters. The PDF of wind power in continuous range (i.e., $v_{\text {cin }}<$ $v \leq v_{r}$ ) is represented by Cui et al. (2020).

$f\left(P_{W}\right)=\frac{k\left(v_{r}-v_{c i n}\right)}{c^{k} P_{W}^{r}}\left(v_{c i n}+\frac{P_{W}}{P_{W}^{r}}\left(v_{r}-v_{c i n}\right)^{k-1}\right) e^{-\left(\frac{v_{\operatorname{cin}}+\frac{P_{W}}{P_{W}^{r}}\left(v_{r}-v_{c i n}\right)}{c}\right)^{k}}$

The PDF of wind power at $P_{W}=0$ is

$f\left(P_{W}=0\right)=f\left(v<v_{i}\right)+f\left(v \geq v_{\text {cout }}\right)=F_{W}\left(v_{i}\right)+\left(1-F_{W}\left(v_{\text {cout }}\right)\right)=1-e^{-\left(\frac{v_{i}}{c}\right)^{k}}+$ $e^{-\left(\frac{v_{\text {cout }}}{c}\right)^{k}}$

The PDF of wind power at $P_{W}=P_{W}^{r}$ is

$f\left(P_{W}=P_{W}^{r}\right)=f\left(v_{r}<v<v_{\text {cout }}\right)=F_{W}\left(v_{\text {cout }}\right)-F_{W}\left(v_{r}\right)=e^{-\left(\frac{v_{r}}{c}\right)^{k}}+e^{-\left(\frac{v_{\text {cout }}}{c}\right)^{k}}$

\subsection{Modeling of Solar PV Generation}

Power generation from the solar PV units depend mainly on solar irradiation $(\mathrm{G})$ and temperature (T) Lee et al. (2015). The amount of solar PV power produced for particular solar irradiation can be expressed as Samman et al. (2020).

$P_{P V}=\left(N_{P V}^{\bmod } \times V \times I \times F F\right)$ 
Characteristics of voltage and current a solar PV unit in terms of ambient temperature $\left(T_{A}\right)$ and solar irradiance $(\mathrm{G})$ are expressed as Altun et al. (2020).

$$
\begin{aligned}
& V=V_{o c}-\left(K_{v} \times T_{c}\right) \\
& I=G\left[I_{s c}+K_{i}\left(T_{c}-25\right)\right] \\
& T_{C}=T_{A}+G\left(\frac{T_{N O T}-20}{0.8}\right) \\
& F F=\frac{V_{M P P} I_{M P P}}{V_{o c} I_{S C}}
\end{aligned}
$$

$T_{A}$ is ambient temperature, $N_{P V}^{\text {mod }}$ is the number of solar PV modules, $F F$ is the fill factor, $T_{C}$ is cell temperature. $V_{M P P}$ and $I_{M P P}$ are maximum power point voltage and current, $V_{o c}$ is opencircuit voltage, $I_{S C}$ is short-circuit current. $K_{v}$ and $K_{i}$ are the temperature coefficient of voltage and current.

Historical data of solar irradiation is used to determine the irradiation probability distribution. In this work, Beta PDF is used, which provides accurate modeling compared to logarithmic and gamma distribution functions. This Beta PDF can be expressed as Huang et al. (2016).

$f(G)=\frac{\Gamma(\alpha+\beta)}{\Gamma(\alpha) \Gamma(\beta)} G^{(\alpha-1)}(1-G)^{(\beta-1)}$

where, $\alpha$ and $\beta$ are the shape parameters and they can be calculated by mean and standard deviation of solar irradiance $(\mathrm{G})$ at a particular time.

\subsection{Load Model of EVCS}

The demand of EVCS mostly depends on number of EVs connected during vehicle-to-grid (V2G) and grid-to-vehicle $(\mathrm{G} 2 \mathrm{~V})$ modes, and the charging and discharging rates and efficiencies. This load demand of EVCS can be expressed as Diaz et al. (2018).

$P_{D}^{E V C S}=\left(N_{E V} P_{G 2 V} \eta_{C} R_{c}\right)-\left(N_{E V} P_{V 2 G} \eta_{d} R_{d}\right)$

$R_{c}$ and $R_{d}$ are the rates of charging and discharging; $\eta_{C}$ and $\eta_{d}$ are charging and discharging efficiencies; $N_{E V}$ is the number of EVs; $P_{G 2 V}$ and $P_{V 2 G}$ are the active powers from G2V and V2G, respectively.

\subsection{Load Modeling}

In this work, the exponential model is selected for modeling the load of DS and EVCSs. The real and reactive demands at any bus i can be related to bus voltage $\left(V_{i}\right)$, using the exponential function, and they are expressed as Lee et al. (2015).

$$
\begin{aligned}
& P_{D i}^{n e w}=P_{D i}\left(\frac{V_{i}}{V_{i}^{0}}\right)^{n p}+P_{D i}^{E V C S}\left(\frac{V_{i}}{V_{i}^{0}}\right)^{n p} \\
& Q_{D i}^{n e w}=Q_{D i}\left(\frac{V_{i}}{V_{i}^{0}}\right)^{n q}+Q_{D i}^{E V C S}\left(\frac{V_{i}}{V_{i}^{0}}\right)^{n q}
\end{aligned}
$$

$P_{D i}^{n e w}, Q_{D i}^{n e w}$ are real and reactive loads at bus i after voltage-dependent/exponential load modeling. $P_{D i}$ and $Q_{D i}$ are real and reactive power demands connected at bus i. $n p$ and $n q$ are 
voltage exponents. $V_{i}^{0}, V_{i}$ are nominal and actual bus voltages.

\section{Mathematical Modeling of ONR}

In this work, distribution load flow is formulated by considering bus-current-injection to branchcurrent and branch-current to bus-voltage matrices. A three-phase transformer is modeled and it is incorporated into the load flow method. Here, ONR/FRC problem is solved by considering total cost and power loss minimizations as objectives.

\subsection{Total Cost (TC) Minimization}

Mminimization of TC objective is formulated by considering the feeder power cost, solar PV, and power from WEG costs. This TC objective can be expressed by Samman et al. (2020).

Minimize,

$T C=\sum_{i=1}^{N_{F}}\left(C_{i} \times P_{i}\right)+\sum_{j=1}^{N_{W}}\left(C_{W j} \times P_{W j}\right)+\sum_{k=1}^{N_{P V}}\left(C_{P V k} \times P_{P V k}\right)$

$N_{F}, N_{W}$ and $N_{P V}$ are the number of feeders, WEGs, and solar PV modules. $P_{i}, P_{W j}$ and $P_{P V k}$ are power output from the feeders, WEGs, and solar PV modules, respectively. $C_{i}, C_{W j}$ and $C_{P V k}$ are cost coefficients of scheduled powers from feeders, WEGs, and solar units, respectively.

\subsection{Power Loss Minimization}

Generally, the distribution systems (DSs) have a high resistance to reactance ratio and therefore they have higher losses. The single line diagram (SLD) of DS with one feeder has been depicted in Figure 1.

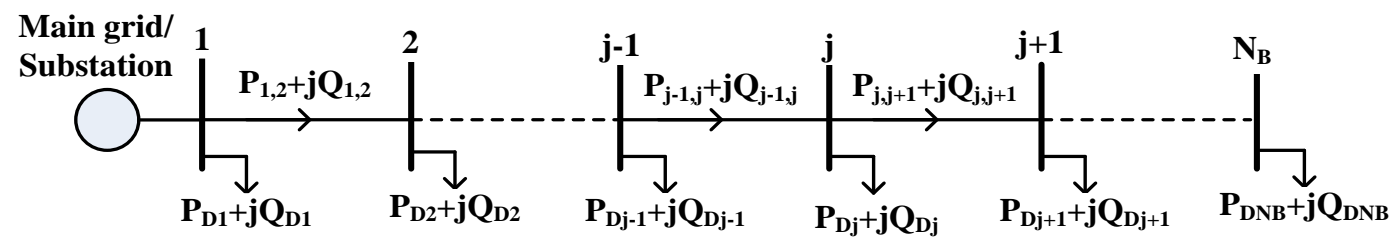

Figure 1. SLD of the distribution system (DS).

Active power loss in a line connected between buses $j$ and $(j+1)$ is calculated as,

$P_{(j, j+1)}^{l o s s}=\left(\frac{P_{(j, j+1)}^{2}+Q_{(j, j+1)}^{2}}{\left|V_{j}\right|^{2}}\right) R_{(j, j+1)}$

Reactive power loss in a line connected between buses $j$ and $(j+1)$ is calculated as Altun et al. (2020).

$Q_{(j, j+1)}^{l o s s}=\left(\frac{P_{(j, j+1)}^{2}+Q_{(j, j+1)}^{2}}{\left|V_{j}\right|^{2}}\right) X_{(j, j+1)}$

Total power loss $\left(P_{T}^{\text {loss }}\right)$ is calculated as,

$P_{T}^{\text {loss }}=\sum_{l=1}^{N_{l}} P_{\text {loss }, l}$ 
$N_{l}$ is the number of lines in DS. The loss minimization objective is expressed as Huang et al. (2016).

minimize $\quad P_{T}^{\text {loss }}$

The above two objective functions in equations (15) and (19) are optimized subject to following constraints.

\subsection{ONR/FRC: Constraints}

The power balance constraint satisfies the sum of total power output from substation, WEGs, solar PV units equal to the sum of total power demand, total losses, and a load of EV charging stations (EVCS). This constraint can be expressed as Cui et al. (2020).

$\sum_{i=1}^{N_{F}} P_{i}+\sum_{j=1}^{N_{W}} P_{W j}+\sum_{k=1}^{N_{P V}} P_{P V k}=P_{D}^{T}+P_{T}^{\text {loss }}+P_{D}^{E V C S}$

where, $P_{D}^{T}$ is total demand in the distribution system.

Radiality constraint: Radial structure of DS can be retained by changing status of tie and sectional switches in the proposed ONR problem Samman et al. (2020).

Each bus voltage must be within lower and upper limits, and it is represented by,

$V_{b}^{\min } \leq V_{b} \leq V_{b}^{\max } \quad b=1,2, \ldots, N_{B}$

Active power in the feeder is restricted by,

$P_{i} \leq P_{i}^{\max } \quad i=1,2, \ldots, N_{F}$

Active power of WEG is restricted by,

$P_{W j} \leq P_{W j}^{\max } \quad j=1,2, \ldots, N_{W}$

Active power of solar PV module is restricted by,

$P_{P V k} \leq P_{P V k}^{\max } \quad k=1,2, \ldots, N_{P V}$

Substation thermal limit is restricted by Samman et al. (2020),

$S_{S S} \leq S_{S S}^{\max }$

Feeder thermal limit is restricted by,

$I_{f} \leq I_{f}^{\max }$

Here, the single objective based FRC/ONR is solved by crow search algorithm (CSA) (Hussien et al., 2020; Meraihi et al., 2020) and multi-objective based ONR is solved by MO-CSA (Nobahari and Bighashdel, 2017). Reader can refer Rizk-Allah et al. (2020) and Movahediyan and Askarzadeh (2018) for the description of CSA and MO-CSA.

\section{Results and Discussion}

The ONR problem is implemented on 17 bus unbalanced and balanced DSs (Raju and Bijwe, 2008). This test system has 17 buses, 19 lines, and 3 transformers (of rating $115 \mathrm{kV} / 13.2 \mathrm{kV}$ ) with delta-Yg connection. These 3 transformers have a leakage impedance of $(0.01+\mathrm{j} 0.05)$ p.u. (Salkuti, 2021) and they are placed in the lines 1, 2 and 3. In this work, the active power exponent (np) is selected as 1 and the reactive power exponent (nq) is selected as 2. The SLD of 17 bus DS 
is shown in Figure 2. In this test system, it is presumed that one wind farm is located at bus 14, one solar PV plant is located at bus 9, and two EVCSs are located on buses 2 and 15 with multiple charging points. Parameters related to solar PV plant are: rated capacity is $2 \mathrm{MW}$, nominal cell operating temperature is $43^{\circ} \mathrm{C}$, open-circuit voltage $\left(V_{o c}\right)$ is $36.96 \mathrm{~V}$, short-circuit current $\left(I_{s c}\right)$ is $8.38 \mathrm{~A}$, voltage at MPP is $28.36 \mathrm{~V}$ and current at MPP is $7.76 \mathrm{~A}$. The rated capacity of WEG is $2 \mathrm{MW}$. It is considered that substation at bus 1 maintains a constant voltage of 1.0 p.u. and the voltage at each bus is restricted between 0.95 p.u. and 1.05 p.u.

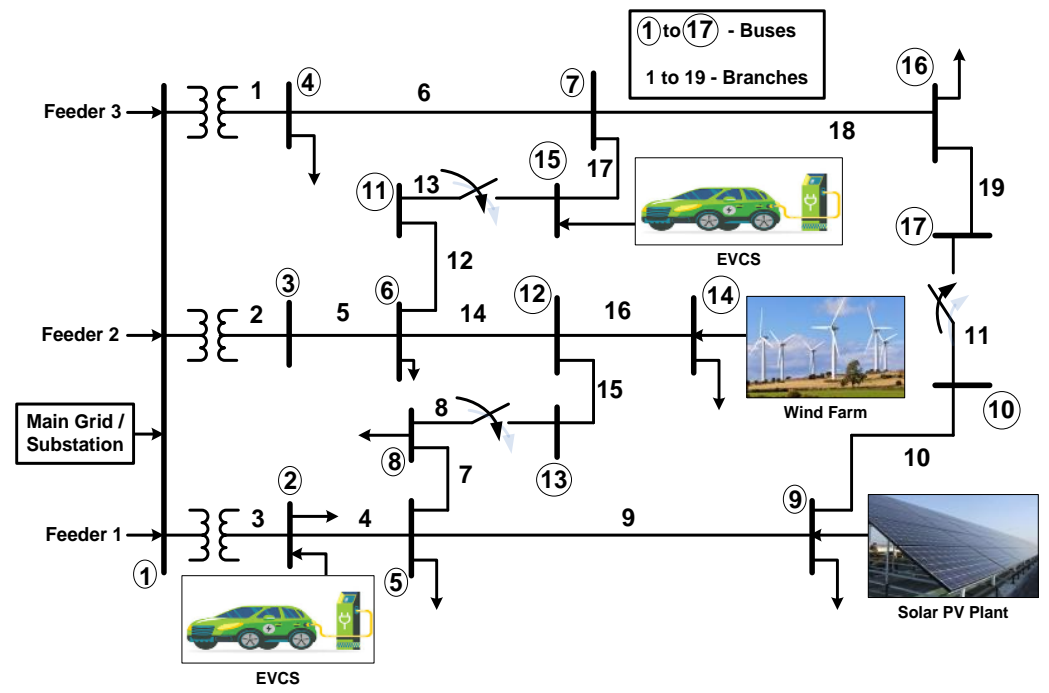

Figure 2. SLD of 17 bus distribution system (DS).

\subsection{Results on 17 Bus Balanced DS}

This section presents the simulation results on 17 bus balanced DS. Power demands, i.e., active and reactive powers considered for this network are 86.10 MW and 51.90 MVAr. In this paper, CSA is used to solve the single-objective optimization (SOO) problems, i.e., power loss and total cost minimization problems, and MO-CSA is used for solving the MO based ONR problem. Table 1 depicts the results for balanced 17 bus DS considering different objective functions. When the minimization of TC is considered at a time then optimum cost obtained is $1965.32 \$ / \mathrm{h}$ and losses are 3.02 MW. The opened lines between the buses after the ONR are 5-8, 5-9, and 611. Optimum power outputs from 3 feeders, one WEG and one solar PV unit are shown in Table 1. When the minimization of loss is optimized alone, the obtained loss is $2.14 \mathrm{MW}$, but cost increased to $2086.19 \$ / \mathrm{h}$.

Table 1. Optimum power outputs for single and multiple objectives of 17 bus balanced DS.

\begin{tabular}{|c|c|c|c|}
\hline & TC Minimization & Power loss Minimization & TC and Loss minimization \\
\hline Feeder 1 power (MW) & 5.32 & 14.07 & 10.89 \\
\hline Feeder 2 power (MW) & 52.06 & 38.44 & 45.39 \\
\hline Feeder 3 power (MW) & 28.59 & 32.82 & 29.16 \\
\hline WEG power (MW) & 1.82 & 1.69 & 1.78 \\
\hline Solar PV power (MW) & 1.85 & 1.74 & 0.52 \\
\hline Load of EVCS (MW) & 0.52 & 0.52 & 2008.04 \\
\hline Cost of operation (\$/h) & 1965.32 & 2086.19 & 2.42 \\
\hline Power loss (MW) & 3.02 & 2.14 & \\
\hline Lines opened (between buses) & \multicolumn{3}{|c|}{$12(6-11), 9(5-9), 7(5-8)$} \\
\hline
\end{tabular}


From the above two SOO problems, it is clear that when cost minimization is optimized separately/alone then power loss has been moved away from its optimum value, and vice versa. Hence, one need to optimize both objectives simultaneously. Therefore, in this work, both the objectives are minimized at a time by using MO-CSA. Figure 3 shows Pareto optimal front (POF), which constitutes 50 compromised solutions of total cost and power loss objectives. Among these 50 compromised solutions, one best-compromised solution has been selected from this Pareto optimal front with the help of fuzzy satisfaction method (Reddy et al., 2011). The trade-off result obtained for this system has cost of $2008.04 \$ / \mathrm{h}$, and power loss of $2.415 \mathrm{MW}$, and this has been depicted in Figure 3. The opened lines between the buses after the ONR are 5-8, 5-9, and 6-11.

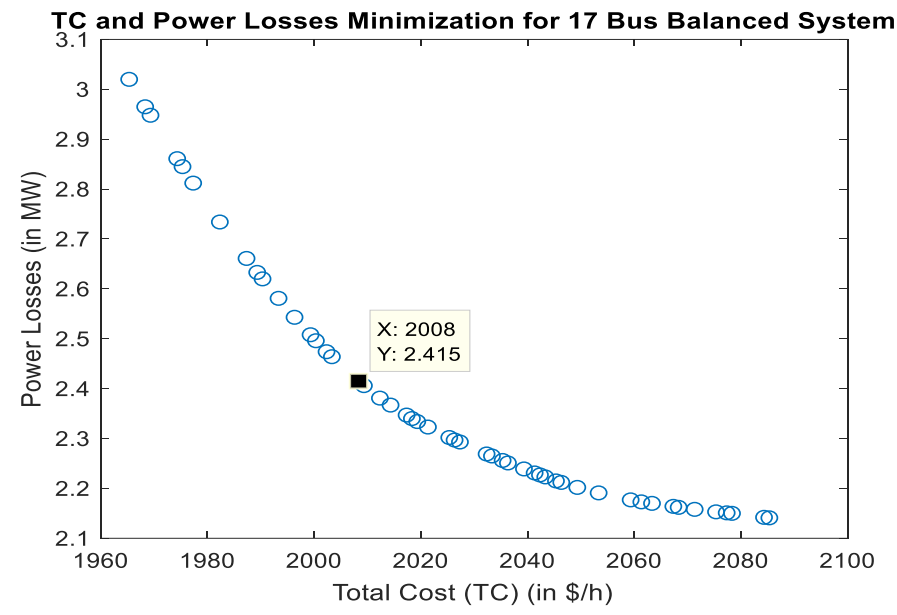

Figure 3. Pareto optimal front (POF) and trade-off solution for balanced 17 bus DS.

\subsection{Results on 17 Bus Unbalanced DS}

This section presents the results on 17 bus unbalanced DS. Power demands, i.e., real and reactive powers considered in this system are 86.96 MW and 52.419 MVAr. Table 2 shows the optimum power outputs for SOO and MOO problem of ONR for unbalanced 17 bus DS considering different objective functions. When cost is optimized at a time then the minimum cost resulted is $2011.07 \$ / \mathrm{h}$ and losses are 3.16 MW. On the other hand, when power loss is minimized alone, then the minimum loss resulted is $2.23 \mathrm{MW}$ but is increased to $2130.14 \$ / \mathrm{h}$. Hence, this work proposes a MOO based ONR problem.

Table 2. Optimum power outputs for single and multiple objectives of 17 bus unbalanced DS.

\begin{tabular}{|c|c|c|c|}
\hline & TC Minimization & Power loss Minimization & TC and Loss minimization \\
\hline Feeder 1 power (MW) & 15.38 & 4.09 & 12.58 \\
\hline Feeder 2 power (MW) & 43.50 & 50.42 & 43.39 \\
\hline Feeder 3 power (MW) & 28.13 & 31.65 & 30.32 \\
\hline WEG power (MW) & 1.84 & 1.80 & 1.81 \\
\hline Solar PV power (MW) & 1.85 & 1.76 & 1.80 \\
\hline Load of EVCS (MW) & 0.58 & 0.58 & 0.58 \\
\hline Cost of operation (\$/h) & 2011.07 & 2130.14 & 2.41 \\
\hline Power loss (MW) & 3.16 & 2.23 & 2.066 .0 \\
\hline Lines opened (between buses) & \multicolumn{2}{|c|}{$8(8-13), 12(6-11), 9(5-9)$} & \\
\hline
\end{tabular}


From the above two SOO problems, it is clear that when total cost is minimized independently then power loss is moved away from its optimum, and vice versa. Therefore, there is a requirement for optimizing both objectives simultaneously. Figure 4 shows the POF, which constitutes 50 compromised solutions of total cost and power loss objectives. Among these 50 compromised solutions, one best-compromised solution has been selected from this Pareto optimal front with the help of fuzzy satisfaction method. The trade-off solution that is resulted for this system has total cost of $2066.02 \$ / \mathrm{h}$, and power loss of $2.407 \mathrm{MW}$, and this has been depicted in Figure 4. The open lines between the buses after the ONR are 5-9, 6-11, and 8-13.

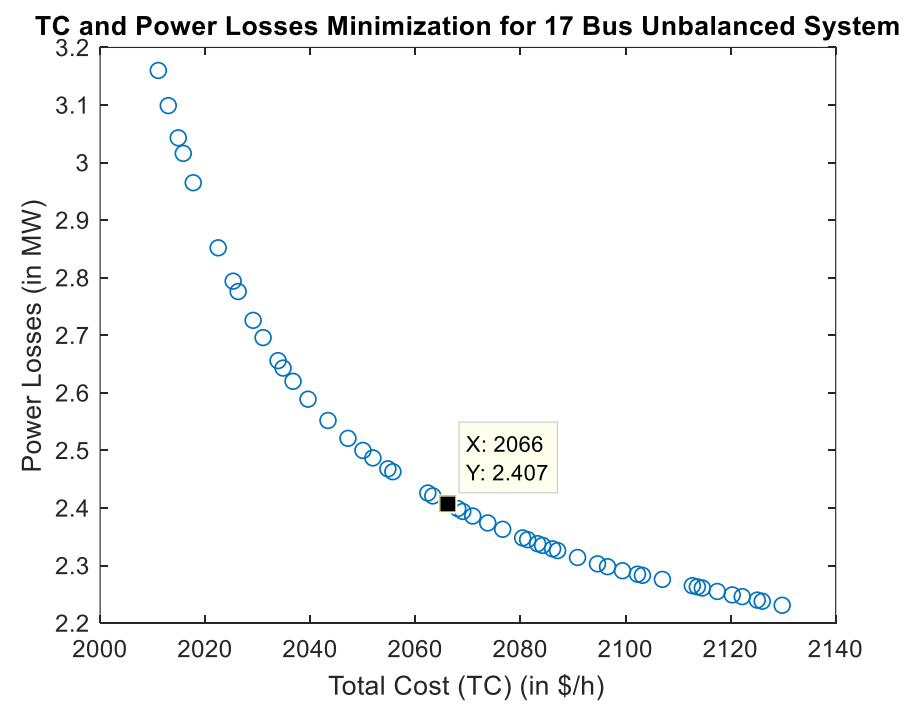

Figure 4. POF and trade-off solution for total cost and power loss minimizations of unbalanced 17 bus DS.

As per the above obtained results, the integration of RESs with ONR leads to reduced power loss, cost and improved voltage levels of the DSs.

\section{Conclusions}

This paper proposes the ONR/FRC problem of the distribution system (DS) with RESs and EVCSs. Generally, DS is an unbalanced system. In unbalanced systems, the three-phase transformers play significant role and it is modeled in this work. The uncertainties related to the RESs are handled by using probability analysis. In this work, wind power uncertainty is handled by using Weibull probability density function (PDF), and solar PV uncertainty is modeled using the Beta PDF. This paper also models the load of EVCSs. The ONR/FRC is used to operate DSs at optimum cost/loss. Therefore in this paper, two objectives (the total cost of operation and power losses) are selected as two objectives functions and they are solved using the evolutionary based algorithms. The multi-objective-based ONR including the switching costs and optimizing the number of switching operations over a period of time is the scope for future research work.

\section{Conflict of Interest}

The authors declare that there is no conflict for this publication.

\section{Acknowledgments}

This research work was funded by "Woosong University's Academic Research Funding -2021". 


\section{References}

Altun, T., Madani, R., Yadav, A.P., Nasir, A., \& Davoudi, A. (2020). Optimal reconfiguration of dc networks. IEEE Transactions on Power Systems, 35(6), 4272-4284.

Amin, A., Tareen, W.U.K., Usman, M., Memon, K.A., Horan, B., Mahmood, A., \& Mekhilef, S. (2020). An integrated approach to optimal charging scheduling of electric vehicles integrated with improved medium-voltage network reconfiguration for power loss minimization. Sustainability, 12(21), 1-15.

Asrari, A., Lotfifard, S., \& Payam, M.S. (2016). Pareto dominance-based multiobjective optimization method for distribution network reconfiguration. IEEE Transactions on Smart Grid, 7(3), 1401-1410.

Babu, P.V.K., \& Swarnasri, K. (2020). Multi-objective optimal allocation of electric vehicle charging stations in radial distribution system using teaching learning based optimization. International Journal of Renewable Energy Research, 10(1), 366-377.

Cui, Z., Bai, X., Li, P., Li, B., Cheng, J., Su, X., \& Zheng, Y. (2020). Optimal strategies for distribution network reconfiguration considering uncertain wind power. CSEE Journal of Power and Energy Systems, 6(3), 662-671.

Diaz, P., Pérez-Cisneros, M., Cuevas, E., Avalos, O., Gálvez, J., Hinojosa, S., \& Zaldivar, D. (2018). An improved crow search algorithm applied to energy problems. Energies, 11(3), 1-22.

Fu, Y.Y., \& Chiang, H.D. (2018). Toward optimal multiperiod network reconfiguration for increasing the hosting capacity of distribution networks. IEEE Transactions on Power Delivery, 33(5), 2294-2304.

Gangwar, P., Mallick, A., Chakrabarti, S., \& Singh, S.N. (2020). Short-term forecasting-based network reconfiguration for unbalanced distribution systems with distributed generators. IEEE Transactions on Industrial Informatics, 16(7), 4378-4389.

Huang, S., Wu, Q., Cheng, L., \& Liu, Z. (2016). Optimal reconfiguration-based dynamic tariff for congestion management and line loss reduction in distribution networks. IEEE Transactions on Smart Grid, 7(3), 1295-1303.

Huang, Z., Fang, B., \& Deng, J. (2020). Multi-objective optimization strategy for distribution network considering V2G-enabled electric vehicles in building integrated energy system. Protection and Control of Modern Power Systems, 5(7), 1-8.

Hussien, A.G., Amin, M., Wang, M., Liang, G., Alsanad, A., Gumaei, A., \& Chen, H. (2020). Crow search algorithm: theory, recent advances, and applications. IEEE Access, 8, 173548-173565.

Jung, C.M., \& Salkuti, S.R. (2020). Optimal wind-thermal coordination scheduling considering reserve requirement. International Journal of Mathematical, Engineering and Management Sciences, 5(1), 108-119.

Kamruzzaman, M.D., Benidris, M., Elsaiah, S., \& Tian, Y. (2020). A method for maximizing the hosting capacity to electric vehicles using feeder reconfiguration. In 2020 IEEE Power \& Energy Society General Meeting (PESGM) (pp. 1-5). IEEE. Montreal, QC, Canada.

Kavousi-Fard, A., Niknam, T., \& Fotuhi-Firuzabad, M. (2015). Stochastic reconfiguration and optimal coordination of $\mathrm{V} 2 \mathrm{G}$ plug-in electric vehicles considering correlated wind power generation. IEEE Transactions on Sustainable Energy, 6(3), 822-830.

Kavousi-Fard, A., Rostami, M.A., \& Niknam, T. (2015). Reliability-oriented reconfiguration of vehicle-togrid networks. IEEE Transactions on Industrial Informatics, 11(3), 682-691.

Kianmehr, E., Nikkhah, S., Vahidinasab, V., Giaouris, D., \& Taylor, P.C. (2019). A resilience-based architecture for joint distributed energy resources allocation and hourly network reconfiguration. IEEE Transactions on Industrial Informatics, 15(10), 5444-5455. 
Koutsoukis, N.C., Siagkas, D.O., Georgilakis, P.S., \& Hatziargyriou, N.D. (2017). Online reconfiguration of active distribution networks for maximum integration of distributed generation. IEEE Transactions on Automation Science and Engineering, 14(2), 437-448.

Lee, C., Liu, C., Mehrotra, S., \& Bie, Z. (2015). Robust distribution network reconfiguration. IEEE Transactions on Smart Grid, 6(2), 836-842.

Li, Z., Jazebi, S., \& León, F. (2017). Determination of the optimal switching frequency for distribution system reconfiguration. IEEE Transactions on Power Delivery, 32(4), 2060-2069.

Liu, Y., Li, J., \& Wu, L. (2019). Coordinated optimal network reconfiguration and voltage regulator/der control for unbalanced distribution systems. IEEE Transactions on Smart Grid, 10(3), 2912-2922.

Meraihi, Y., Gabis, A.B., Ramdane-Cherif, A., \& Acheli, D. (2020). A comprehensive survey of crow search algorithm and its applications. Artificial Intelligence Review, 54, 2669-2716.

Movahediyan, Z., \& Askarzadeh, A. (2018). Multi-objective optimization framework of a photovoltaicdiesel generator hybrid energy system considering operating reserve. Sustainable Cities and Society, $41,1-12$.

Mozafar, M.R., Moradi, M.H., \& Amini, M.H. (2017). A simultaneous approach for optimal allocation of renewable energy sources and electric vehicle charging stations in smart grids based on improved GAPSO algorithm. Sustainable Cities and Society, 32, 627-637.

Naidu, K., Muhammad, M.A., Mokhlis, H., Sufyan, M., \& Amin, A. (2019). Optimal coordination of EV charging with network reconfiguration. In 2019 AIP Conference Proceedings (Vol. 2129, No. 1, p. 020094). AIP. Jawa Barat, Indonesia.

Nick, M., Cherkaoui, R., \& Paolone, M. (2018). Optimal planning of distributed energy storage systems in active distribution networks embedding grid reconfiguration. IEEE Transactions on Power Systems, 33(2), 1577-1590.

Nobahari, H., \& Bighashdel, A. (2017). MOCSA: a multi-objective crow search algorithm for multiobjective optimization. In $20172^{\text {nd }}$ Conference on Swarm Intelligence and Evolutionary Computation (CSIEC) (pp. 60-65). IEEE. Kerman, Iran.

Pamshetti, V.B., Singh, S., \& Singh, S.P. (2020). Combined impact of network reconfiguration and volt-var control devices on energy savings in the presence of distributed generation. IEEE Systems Journal, 14(1), 995-1006.

Peng, Q., Tang, Y., \& Low, S.H. (2015). Feeder reconfiguration in distribution networks based on convex relaxation of opf. IEEE Transactions on Power Systems, 30(4), 1793-1804.

Raju, G.K.V., \& Bijwe, P.R. (2008). Efficient reconfiguration of balanced and unbalanced distribution systems for loss minimisation. IET Generation, Transmission \& Distribution, 2(1), 7-12.

Reddy, S.S., Abhyankar, A.R., \& Bijwe. P.R. (2011). Reactive power price clearing using multi-objective optimization. Energy, 36(5), 3579-3589.

Rizk-Allah, R.M., Hassanien, A.E., \& Slowik, A. (2020). Multi-objective orthogonal opposition-based crow search algorithm for large-scale multi-objective optimization. Neural Computing and Applications, 32, 13715-13746.

Rostami, M.A., Kavousi-Fard, A., \& Niknam, T. (2015). Expected cost minimization of smart grids with plug-in hybrid electric vehicles using optimal distribution feeder reconfiguration. IEEE Transactions on Industrial Informatics, 11(2), 388-397.

Sadeghian, O., Nazari-Heris, M., Abapoue, M., Taheri, S.S., \& Zare, K. (2019). Improving reliability of distribution networks using plug-in electric vehicles and demand response. Journal of Modern Power Systems and Clean Energy, 7(5), 1189-1199. 
Salkuti, S.R. (2021). Multi-objective based optimal network reconfiguration using crow search algorithm. International Journal of Advanced Computer Science and Applications, 12(3), 86-95.

Samman, M.A., Mokhlis, H., Mansor, N.N., Mohamad, H., Suyono, H., \& Sapari, N.M. (2020). Fast optimal network reconfiguration with guided initialization based on a simplified network approach. IEEE Access, 8, 11948-11963.

Wu, H., Dong, P., \& Liu, M. (2020). Distribution network reconfiguration for loss reduction and voltage stability with random fuzzy uncertainties of renewable energy generation and load. IEEE Transactions on Industrial Informatics, 16(9), 5655-5666. 